\title{
Variability in the effects of prehospital advanced airway management on outcomes of patients with out-of-hospital cardiac arrest
}

\section{Young Seok Oh', Ki Ok Ahn', Sang Do Shin ${ }^{2,3}$, Kentaro Kagino ${ }^{4}$, Tatsuya Nishiuchi ${ }^{5}$, Matthew Ma ${ }^{6}$, Patrick Ko ${ }^{6,7}$, Marcus Eng Hock Ong ${ }^{8,9}, \mathrm{Ng}$ Yih Yng ${ }^{10,11}$, Benjamin Leong ${ }^{12}$}

\author{
'Department of Emergency Medicine, Myongji Hospital, Hanyang University College of Medicine, Goyang, \\ Korea \\ ${ }^{2}$ Department of Emergency Medicine, Seoul National University College of Medicine, Seoul, Korea \\ ${ }^{3}$ Laboratory of Emergency Medical Services, Seoul National University Hospital Biomedical Research \\ Institute, Seoul, Korea \\ ${ }^{4}$ Department of Emergency and Critical Care Medicine, Kansai Medical University, Osaka, Japan \\ ${ }^{5}$ Department of Emergency and General Medicine, Amagasaki General Medical Center, Osaka, Japan \\ ${ }^{6}$ Department of Emergency Medicine, National Taiwan University Hospital, National Taiwan University, \\ Taipei, Taiwan \\ ${ }^{7}$ Institute of Epidemiology and Preventive Medicine, College of Public Health, National Taiwan University, \\ Taipei, Taiwan \\ ${ }^{8}$ Department of Emergency Medicine, Singapore General Hospital, Singapore \\ ${ }^{9}$ Health Services and Systems Research, Duke-NUS Graduate Medical School, Singapore \\ ${ }^{10}$ Emergency Department, Tan Tock Seng Hospital, Singapore \\ ${ }^{11}$ Home Team Medical Services Division, Ministry of Home Affairs, Singapore \\ ${ }^{12}$ Emergency Medicine Department, National University Hospital, Singapore
}

Objective To investigate variations in the effects of prehospital advanced airway management (AAM) on outcomes of out-of-hospital cardiac arrest (OHCA) patients according to regional emergency medical service (EMS) systems in four Asian cities.

Methods We enrolled adult patients with EMS-treated OHCA of presumed cardiac origin between 2012 and 2014 from Osaka (Japan), Seoul (Republic of Korea), Singapore (Singapore), and Taipei (Taiwan). The main exposure variable was prehospital AAM. The primary endpoint was neurological recovery. We compared outcomes between the prehospital AAM and non-AAM groups using multivariable logistic regression with an interaction term between prehospital AAM and the four Asian cities.

Results A total of 16,510 patients were included in the final analyses. The rates of prehospital AAM varied among Osaka, Seoul, Singapore, and Taipei $(65.0 \%, 19.2 \%$, 84.9\%, and 34.1\%, respectively). The non-AAM group showed better outcomes than the AAM group (adjusted odds ratio [aOR] for neurological recovery $0.30 ; 95 \%$ confidence interval [Cl], 0.24-0.38]). In the interaction model for neurological recovery, the aORs for AAM in Osaka and Singapore were 0.12 $(95 \% \mathrm{Cl}, 0.06-0.26)$ and $0.21(95 \% \mathrm{Cl}, 0.16-0.28)$, respectively. In Seoul and Taipei, the association between prehospital AAM and neurological recovery was not significant (aOR $0.58[95 \% \mathrm{Cl}$, $0.31-1.10]$ and 0.79 [95\% $\mathrm{Cl}, 0.52-1.20]$, respectively). The interaction between prehospital $A A M$ and region was significant $(P=0.01)$.

Conclusion The effects of prehospital AAM on outcomes of OHCA patients differed according to regional variability in the EMS systems.

Keywords Out-of-hospital cardiac arrest; Airway management; Emergency medical services
elSSN: 2383-4625

Received: 11 March 2019

Revised: 11 June 2019

Accepted: 8 July 2019

Correspondence to: Ki Ok Ahn Department of Emergency Medicine, Myongji Hospital, Hanyang University College of Medicine, 55 Hwasu-ro 14beon-gil, Deokyang-gu, Goyang 10475, Korea

E-mail: arendt75@hanyang.ac.kr ORCID

http://orcid.org/0000-0002-8446-3269

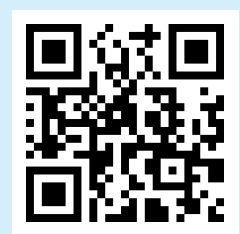

How to cite this article:

Oh YS, Ahn KO, Shin SD, Kagino K, Nishiuchi T, Ma M, Ko P, Ong ME, Yng NY, Leong B. Variability in the effects of prehospital advanced airway management on outcomes of patients with out-of-hospital cardiac arrest. Clin Exp Emerg Med 2020;7(2):95106.

This is an Open Access article distributed under the terms of the Creative Commons Attribution Non-Commercial License (https:// creativecommons.org/licenses/by-nc/4.0/). 


Capsule
$\begin{aligned} & \text { What is already known } \\ & \text { The effects of prehospital advanced airway management (AAM) on outcomes of patients with out-of-hospital cardiac } \\ & \text { arrest is controversial. The multifaceted, unmeasured factors such as the timing of prehospital AAM or the proficiency } \\ & \text { of emergency medical service personnel lead to inconsistent effects of prehospital AAM. }\end{aligned}$
$\begin{aligned} & \text { What is new in the current study } \\ & \text { There was regional variation in the effects of prehospital AAM on outcomes among patients with out-of-hospital car- } \\ & \text { diac arrest. }\end{aligned}$

\section{INTRODUCTION}

Sudden cardiac arrest is a major cause of death worldwide. In the United States, 38 people experience out-of-hospital cardiac arrest (OHCA) every hour. ${ }^{1}$ Although the outcomes of patients with OHCA have improved in recent decades due to the continuous efforts of emergency medical services (EMSs) in enhancing the "chain of survival," regional variability exists in the rates of survival and favorable neurological outcomes. ${ }^{2}$

Advanced airway management (AAM) is crucial for oxygen delivery in patients with OHCA. However, recent studies have shown conflicting effects of prehospital AAM on outcomes. ${ }^{3,4}$ These inconsistent effects could be attributed to multifaceted, unmeasured factors such as timing of prehospital AAM or the proficiency of EMS personnel. In prehospital settings, it is challenging to measure and document the exact time of prehospital AAM performance. The definitions of proficiency levels in prehospital AAM also remain controversial.

To evaluate the comprehensive effects of unmeasured prehospital factors, the regional EMS system can be used as a unit of analysis. ${ }^{5}$ The effects of AAM may vary by regional EMS system due to differences in the AAM protocols or proficiency levels of EMS providers. ${ }^{6}$ Gaining a better understanding of regional variability and its effects on AAM may aid EMS directors and stakeholders in the development of prehospital AAM protocols and training programs that are optimized for individual regional EMS systems. Obtaining further information on this variability may also inform the magnitude of opportunities for region-wide outcome improvements in patients with OHCA.

This study investigated and compared the effects of prehospital AAM on outcomes within four different regional EMS systems. We hypothesized that the effect of prehospital AAM is constant across regional EMS systems.

\section{METHODS}

\section{Study design and setting}

This study was a secondary analysis of data from a prospective observational study of OHCA patients in four cities: Osaka (Japan), Seoul (Republic of Korea), Singapore (Singapore), and Taipei (Taiwan). We used data from the Pan-Asian Resuscitation Outcomes Study (PAROS) clinical research network.?

The EMS systems of the four Asian cities covered metropolitan cities with populations ranging from 2.5 (Osaka) to 9.9 (Seoul) million people. All four EMS systems were tax-based and operated by fire departments. The EMS systems varied between the study regions. Two EMS systems are single-tiered, while the Singapore and Taipei EMS systems are multi-tiered. The highest emergency medicine technician (EMT) level in three regions is equivalent to EMT-intermediate (EMT-I) level in the United States. In Taipei city, paramedics constitute the highest service level of EMTs. ${ }^{8}$ In addition, the prehospital AAM protocols also vary between the cities. In two regions (Osaka and Seoul), an EMT-I can perform endotracheal intubation (ETI) or supra-glottic airway (SGA) under direct medical oversight. In Singapore, an EMT-I can only perform SGA under indirect medical oversight. In Taipei city, paramedics can perform both ETI and SGA without online medical control.

The annual incidence of EMS-treated OHCA per 100,000 persons ranged from 47.4 (Singapore) to 87.1 (Osaka). ${ }^{9,10}$ The highest rate of survival to discharge and neurologic recovery was demonstrated in Seoul (9.9\% and 3.7\%, respectively). ${ }^{8}$

\section{Data source}

The PAROS was established in 2009 with the aim of understanding the OHCA epidemiology in Asia. The goal of the PAROS is to develop and implement widespread community-based and systemic efforts to improve patient outcomes. EMS-treated OHCA cases involving individuals of all ages were captured regardless of 
the etiology of cardiac arrest (presumed cardiac and non-cardiac etiology). In Singapore and Thailand, non-EMS-treated OHCA cases that presented to the emergency department (ED) were also enrolled in the PAROS dataset. Further details and information on the data quality management processes used in the PAROS project have been presented elsewhere. ${ }^{?}$

\section{Study population}

We included adult (aged over 18 years) patients with EMS-treated OHCA of presumed cardiac origin in Osaka, Seoul, Singapore, and Taipei, who were entered in the PAROS registry between 2012 and 2014. We excluded patients in whom the OHCA was witnessed by EMS personnel and those with unknown information on prehospital AAM performance and outcomes. Cardiopulmonary resuscitation (CPR) was not initiated in all the participating sites if obvious signs of death are observed in prehospital settings. An obvious sign of death was defined as the presence of decapitation, rigor mortis, dependent lividity, and "do not attempt resuscitation (DNR)" orders; patients with obvious signs of death in prehospital settings were excluded from the PAROS dataset. DNR orders are valid in both hospital and prehospital settings in Seoul, Singapore, and Taipei; in Osaka, DNR orders are only valid in hospital settings. Termination of resuscitation was not allowed in all four regional EMS systems."11

This study was approved by all the institutional review boards of the participating study sites. The need for informed consent was waived.

\section{Data collection and variables}

The registry included data on the OHCA patients' socio-demographic characteristics, as well as EMS and ED information based on the Utstein template, such as circumstances of the OHCA (witness status, bystander CPR), electrocardiogram (ECG) rhythm at cardiac arrest, EMS time intervals, emergency care provided by EMSs and in the ED, and patient outcomes. ${ }^{12}$ The initial ECG rhythm at cardiac arrest was assessed by EMS providers and was classified as shockable (ventricular fibrillation and pulseless ventricular tachycardia), non-shockable (asystole, pulseless electrical activity and severe bradycardia in pediatric patients), and unknown. The EMS time intervals included EMS response time and EMS scene time intervals. The EMS response interval was defined as the duration from call to arrival at the scene. The EMS scene time interval was defined as the duration from arrival at the scene to departure. We collected information on the prehospital care provided by the EMS, including airway management. PAROS data included those on hospital care, such as use of targeted temperature management, percutaneous coronary intervention, and extracorporeal membrane oxygenation.

The primary exposure was prehospital AAM, which included all the AAM methods used. We defined AAM as an invasive technique used for airway management, including ETI and all types of SGA. The non-AAM group included patients who underwent a non-invasive technique for airway management, such as use of a bag valve mask, with or without the inclusion of the nasopharyngeal and/or oropharyngeal airways. The primary outcome was neurological recovery at hospital discharge or 30 days after OHCA. Neurologic recovery was defined as a Cerebral Performance Category score of 1 or 2 at discharge. ${ }^{13} \mathrm{~A}$ score of 1 indicates mild neurologic deficit or its absence; 2 , moderate cerebral disability; 3 , severe cerebral disability; 4 , coma or vegetative state; and 5 , brain death. The secondary outcome was survival to discharge.

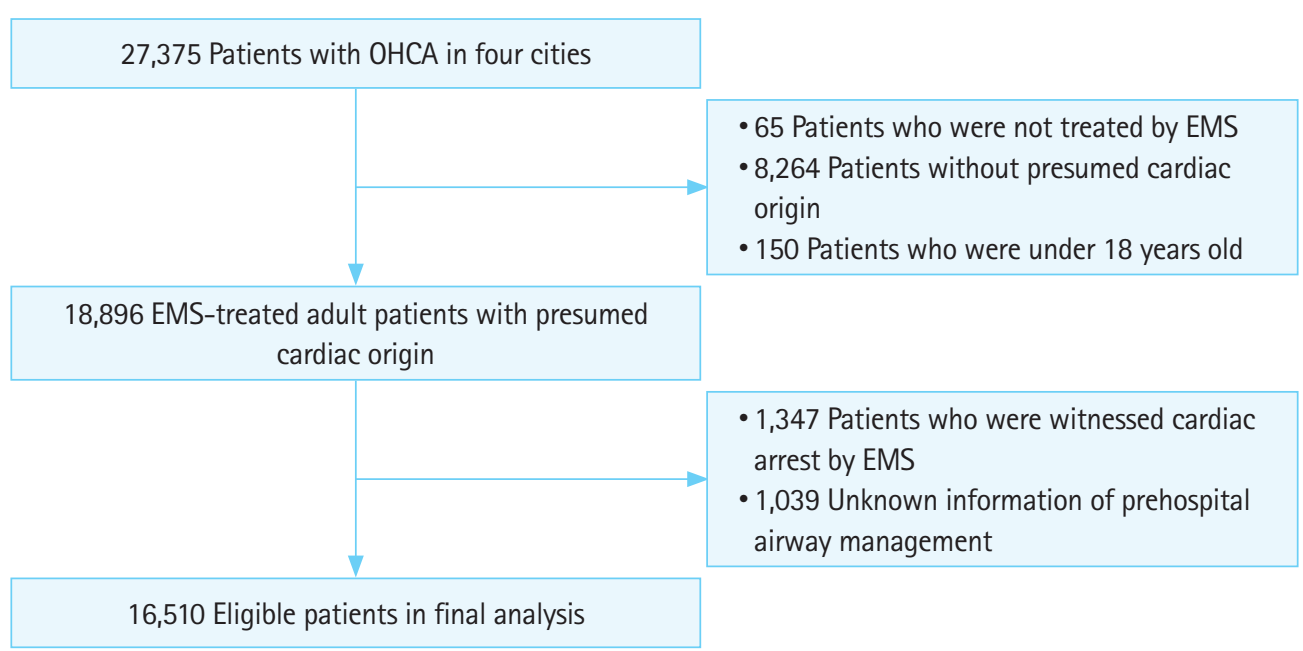

Fig. 1. Flow chart of patient selection and exclusion from the analysis. OHCA, out-of-hospital cardiac arrest; EMS, emergency medical service. 


\section{Statistical analysis}

The demographic findings and outcomes based on prehospital AAM in the four Asian cities were described. Continuous data were reported as medians and interquartile range (IQR) and categorical variables as counts and percentages. Two-sided tests were conducted and $\mathrm{P}<0.05$ was considered statistically significant.

Backward, stepwise multivariable logistic regression analyses were conducted to estimate the effect of prehospital AAM on outcomes. A significance level of 0.1 was required for a variable to remain in the model. The adjusted odds ratios (aORs) and 95\% confidence intervals (Cls) were calculated after adjusting for potential risk factors, such as Utstein variables (age, sex, witness status, bystander CPR, EMS response time, EMS scene time, and prehospital ECG rhythm [shockable vs. non-shockable]), as co- variates. The interactions between prehospital $A A M$ and regional EMS systems that performed prehospital AAM for the effect on outcomes were tested to compare the effect size of prehospital AAM, adjusting for the same co-variables.

\section{RESULTS}

A total of 18,896 adult patients with OHCA of presumed cardiac origin were enrolled in the PAROS registry from the four Asian cities. After the exclusion of 1,347 (7.1\%) patients with OHCA witnessed by EMS providers and 1,039 (5.5\%) of those with unknown information on prehospital airway management, the final analysis included 16,510 patients (Fig. 1).

Table 1 shows the patient characteristics based on prehospital

Table 1. Characteristics of patients with out-of-hospital cardiac arrest according to prehospital advanced airway use

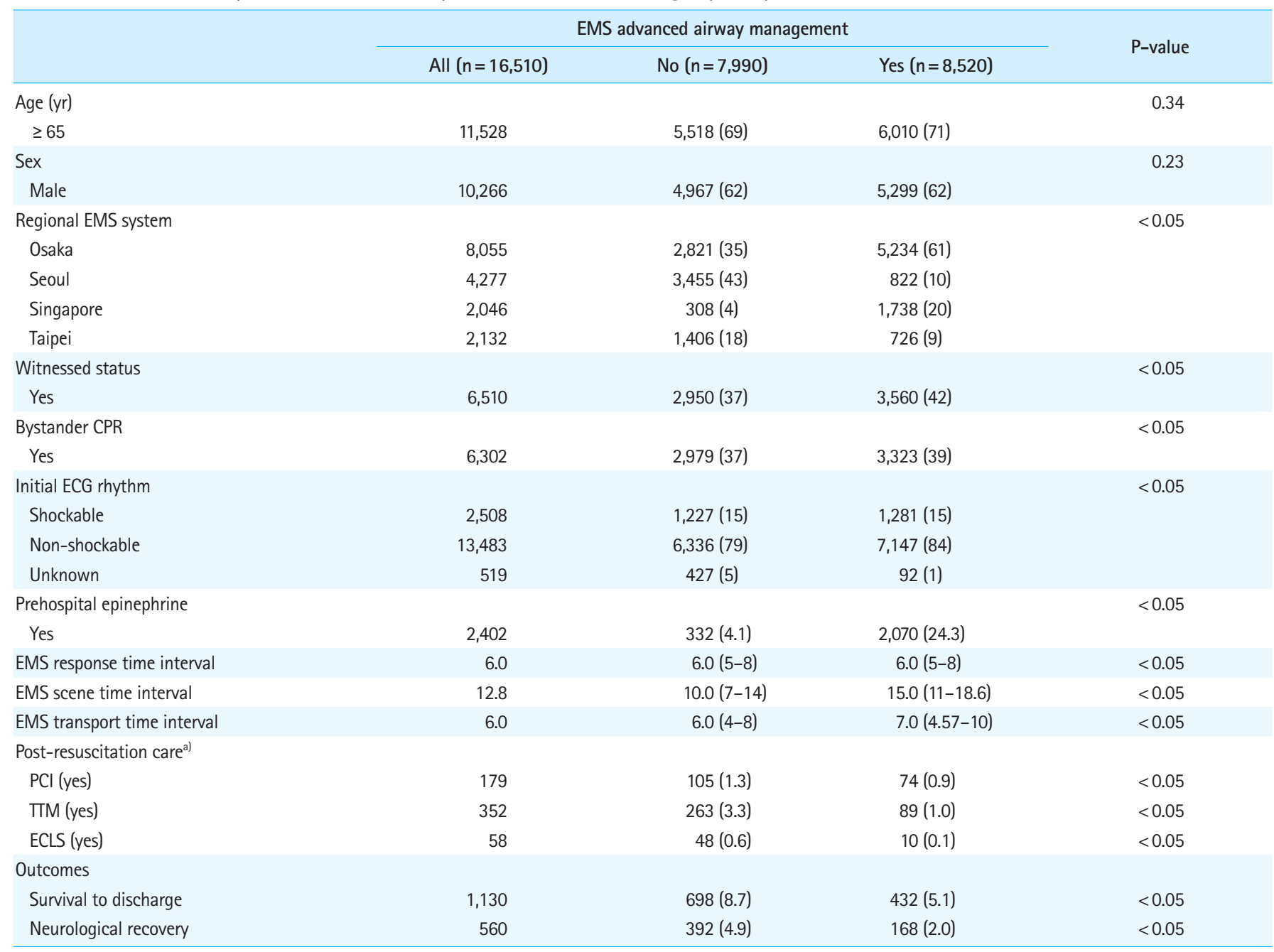

Values are presented as number, number (\%), or median (interquartile range).

EMS, emergency medical services; CPR, cardiopulmonary resuscitation; ECG, electrocardiography; PCl, percutaneous coronary intervention; TTM, targeted temperature management; ECLS, extracorporeal life support.

${ }^{a)}$ Data of post-resuscitation cares was not available in Osaka and Taipei. 
AAM. Prehospital AAM was performed in 52\% of the patients. The rate of prehospital AAM differed significantly across the four regional EMS systems. More than half of the patients with OHCA in Singapore (84\%) and Osaka (65\%) received prehospital AAM. However, the frequencies of prehospital AAM were relatively low in Taipei (34.1\%) and Seoul (19.2\%). Significant differences in the patients' characteristics and outcomes were observed based on prehospital AAM. The scene time interval was significantly longer in the AAM group than the non-AAM group (median time interval 10 minutes [IOR, 7-14] vs. 15 minutes [IOR, 11-18.5]). The non-AAM group showed better neurological recovery rates than the AAM group ( $4.9 \%$ vs. $2.0 \%, \mathrm{P}<0.01$ ).

Table 2 shows the characteristics of patients with OHCA according to the four regional EMS systems. The rates of prehospital
AAM differed considerably between each site, from $19.2 \%$ to $84 \%$. The neurological recovery rates associated with OHCA also varied, from $1.6 \%$ to $3.8 \%$.

After adjusting for potential covariates, patients who received prehospital AAM had lower odds of neurological recovery (aOR 0.30 [95\% Cl, 0.24-0.38]). A similar result was observed for survival to discharge (aOR 0.58 [95\% Cl, 0.50-0.648]). When the region with the poorest outcomes was used as a reference, the highest aORs of neurological recovery and survival to discharge were observed in Osaka (aOR 3.95 [95\% Cl, 2.64-5.90] and 4.51 [95\% Cl, 3.36-6.05] respectively) (Table 3).

In the interaction model for outcomes, the aORs for prehospital AAM on outcomes differed according to region (Table 4). In Osaka and Singapore, prehospital AAM showed low aORs for neu-

Table 2. Characteristics of patients with out-of-hospital cardiac arrest according to regional emergency medical services system

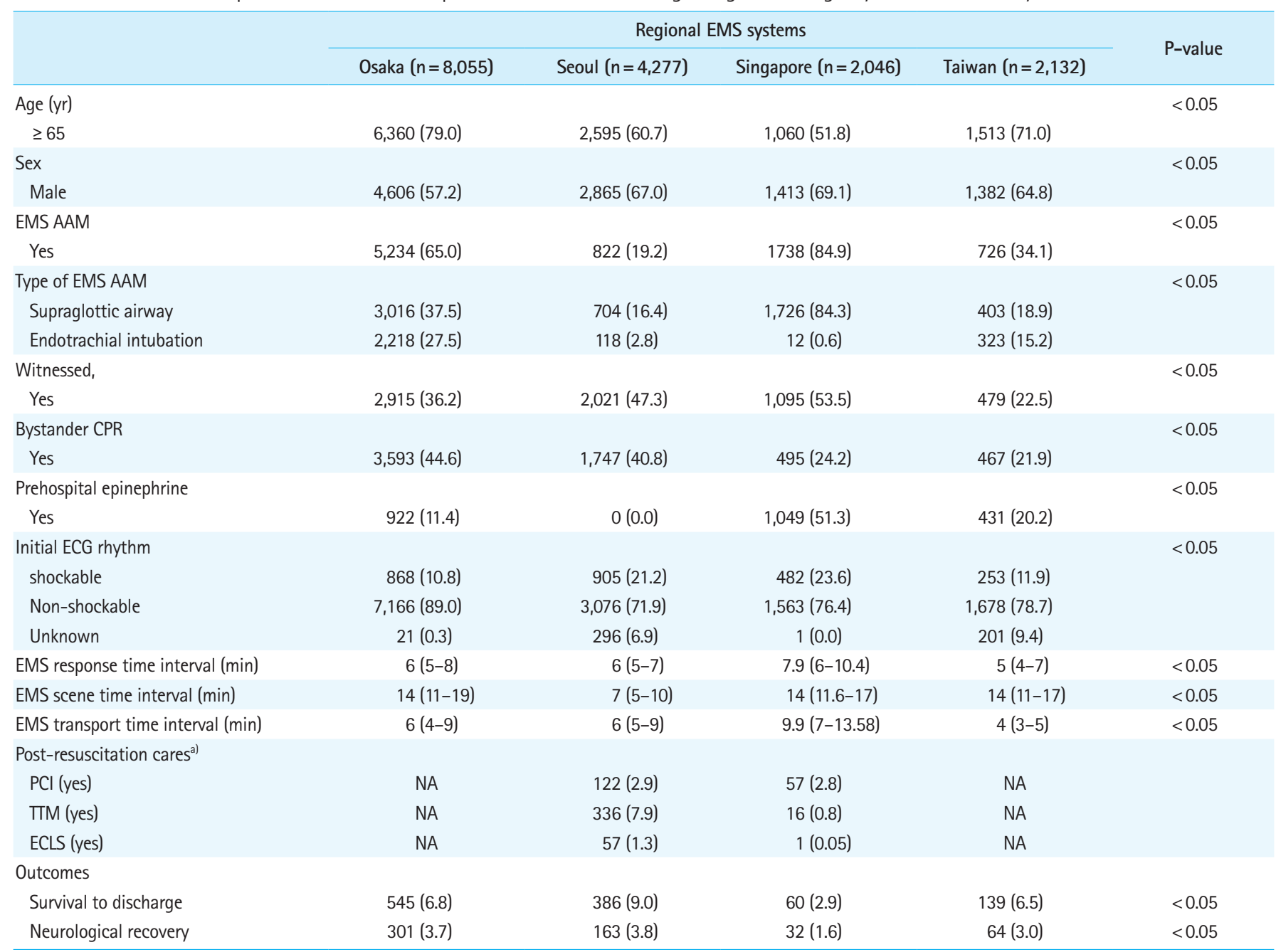

Values are presented as number (\%) or median (interquartile range).

EMS, emergency medical services; AAM, advanced airway management; $C P R$, cardiopulmonary resuscitation; ECG, electrocardiography; $\mathrm{PCl}$, percutaneous coronary intervention; NA, not available; $\Pi M M$, targeted temperature management; ECLS, extracorporeal life support.

${ }^{a}$ Data on post-resuscitation care was not available in Osaka and Taipei. 
Table 3. Multivariable analysis for the effects of prehospital advanced airway management and regional EMS systems on outcomes

\begin{tabular}{|c|c|c|c|c|c|c|}
\hline & Total & Outcomes & \multicolumn{2}{|c|}{ Unadjusted } & \multicolumn{2}{|c|}{ Adjusted } \\
\hline \multicolumn{7}{|c|}{ Survival to discharge } \\
\hline No & 7,990 & $698(8.7)$ & 1.00 & & 1.00 & \\
\hline Yes & 8,520 & $432(5.1)$ & 0.56 & $0.49-0.63$ & 0.58 & $0.50-0.68$ \\
\hline Taipei & 2,132 & $139(6.5)$ & 2.31 & $1.69-3.14$ & 3.42 & $2.42-4.84$ \\
\hline Osaka & 8,055 & $545(6.8)$ & 2.40 & $1.83-3.15$ & 4.51 & $3.36-6.05$ \\
\hline Seoul & 4,277 & $386(9.0)$ & 3.28 & $2.49-4.33$ & 2.24 & $1.63-3.09$ \\
\hline \multicolumn{7}{|c|}{ Neurological recovery } \\
\hline \multicolumn{7}{|c|}{ Prehospital advanced airway management ${ }^{\mathrm{a})}$} \\
\hline Taipei & 2,132 & $64(3.0)$ & 1.95 & $1.27-2.99$ & 1.96 & $1.21-3.18$ \\
\hline Osaka & 8,055 & $301(3.7)$ & 2.44 & $1.69-3.53$ & 3.95 & $2.64-5.90$ \\
\hline Seoul & 4,277 & 163 (3.8) & 2.49 & $1.70-3.66$ & 1.00 & $0.64-1.56$ \\
\hline
\end{tabular}

Values are presented as number or number (\%).

EMS, emergency medical services; $\mathrm{OR}$, odds ratio; $\mathrm{Cl}$, confidence interval.

${ }^{\text {a) }}$ Adjusted to city, sex, age, witness arrest, bystander cardiopulmonary electrocardiography, response time interval, scene time interval. ${ }^{\text {b)} A d j u s t e d ~ t o ~ E M S ~ a d v a n c e d ~ a i r w a y ~}$ management, sex, age, witness arrest, bystander cardiopulmonary resuscitation, initial electrocardiography, response time interval, scene time interval.

Table 4. Effects of prehospital advanced airway management on outcomes in interaction model with regional emergency medical services systems

\begin{tabular}{llll}
\hline Outcomes & & Adjusted OR & $95 \% \mathrm{Cl}$ \\
\hline $\begin{array}{l}\text { Survival to discharge } \\
\text { Interaction }\end{array}$ & AAM(-) & 1 & \\
Singapore & AAM(+) & 0.31 & $0.17-0.58$ \\
Taipei & AAM(+) & 1.04 & $0.69-1.55$ \\
Osaka & AAM(+) & 0.43 & $0.35-0.52$ \\
Seoul & AAM(+) & 0.99 & $0.75-1.30$ \\
Neurological recovery & & & \\
Interaction & AAM(-) & 1 & \\
Singapore & AAM(+) & 0.12 & $0.06-0.26$ \\
Taipei & AAM(+) & 0.58 & $0.31-1.10$ \\
Osaka & AAM(+) & 0.21 & $0.16-0.28$ \\
Seoul & AAM(+) & 0.79 & $0.52-1.20$ \\
\hline
\end{tabular}

$\mathrm{OR}$, odds ratio; $\mathrm{Cl}$, confidence interval; $\mathrm{AAM}$, advanced airway management.

rologic recovery (aOR $0.12[95 \% \mathrm{Cl}, 0.06-0.26]$ and $0.21[95 \% \mathrm{Cl}$, 0.16-0.28], respectively). In contrast, there were no significant associations between prehospital AAM and neurologic recovery (aOR 0.58 [95\% Cl, 0.31-1.10] and 0.79 [95\% Cl, 0.52-1.20]) in Taipei and Seoul. The effect of prehospital AAM on survival across the regions was similar to that observed for survival to discharge (Table 3).

\section{DISCUSSION}

This study aimed to assess regional variations in prehospital AAM use and its effects across four Asian EMS systems. This study highlighted that the effect of prehospital AAM on outcomes varied according to regional EMS systems.

The results of the present study showed the presence of regional variations in prehospital AAM across Asian EMS systems. The highest proportion of prehospital AAM was more than four times that observed in the region with the lowest proportion. Until recently, regional variations in prehospital AAM did not attract the attention of researchers as, in the United States, more than 80\% of OHCA patients received prehospital AAM during the past decades. ${ }^{11}$ As EMS systems have evolved in countries other than those in the West, regional variability in prehospital AAM has also been observed. As regional EMS systems have different infrastructures, each agency should establish an individual strategy for prehospital AAM training and continuous quality assurance. Beyond benchmarking, scientific evidence is also required to provide details for the formulation of protocols for each regional EMS system.

The main result of this study was the observation that regional EMS systems modified the prehospital AAM effect on outcomes of patients with OHCA. Previously, associations between prehospital AAM and outcomes of patients with OHCA were observed in 
several regions. ${ }^{14,15}$ Those results are similar to ours. Several nationwide retrospective observational studies in Japan have reported that prehospital AAM is associated with poor outcomes. ${ }^{15}$ In a Korean nationwide retrospective cohort study, prehospital ETI was not associated with survival. ${ }^{16}$

The mechanisms of the observed regional variability in the effects of prehospital AAM are not clear. Several factors may contribute to these variations: type of AAM; process of AAM; and unmeasured factors, such as proficiency of EMS providers and teamwork among prehospital care providers. There is controversy surrounding the effects of prehospital AAM according to AAM types. A previous meta-analysis of randomized controlled trials (RCTs) reported no significant differences in survival and neurologic outcomes between ETI and SGA. ${ }^{17}$ In this study, the proportion of SGA use was relatively high in Seoul (85.6\%) and Singapore (96.8\%) among patients who received prehospital AAM. However, the effects of prehospital AAM in each region were different. Therefore, regional variations in the effects of prehospital AAM could not be explained by the type of prehospital AAM in each region. In the provision of prehospital AAM, timing is crucial. A recent study reported that early prehospital AAM provision was associated with neurological recovery in patients with OHCA. In their observational study, Izawa et al. ${ }^{18}$ reported resuscitation time bias in early return of spontaneous circulation as a limitation. Another recent RCT did not include information on the timing of prehospital AAM. ${ }^{19}$ The optimal timing for prehospital AAM remains controversial. Although we did not include information on the exact timing of prehospital AAM from PAROS data, we conducted sensitivity analyses for scene time interval. The onscene CPR durations limited by the regional EMS protocols for patients with non-traumatic OHCA are 4 minutes in Osaka and 2 minutes in Singapore and Taipei. ${ }^{19}$ In Seoul, the duration of onscene CPR varies according to response time, ${ }_{11}^{11}$ and the timing of prehospital AAM is recommended prior to departure. The EMS protocols in the other three regions did not include recommendations for the timing of prehospital AAM. When patients achieve early prehospital return of spontaneous circulation, providers may not perform prehospital AAM; thus, the scene time of these patients may be short. This could have resulted in better outcomes in the prehospital non-AAM group. To overcome selection bias, we conducted sensitivity analyses that excluded patients with scene time intervals shorter than 8 minutes. The results of the sensitivity analysis were similar to those of the entire analysis, and demonstrated similar patterns (Appendices 1, 2). After the exclusion of patients with scene time intervals shorter than 8 minutes, prehospital AAM was negatively associated with neurologic recovery and survival to discharge $(\mathrm{aOR} 0.25[95 \% \mathrm{Cl}, 0.20-0.33]$ and 0.53
[95\% Cl, 0.44-0.63], respectively) (Appendix 3). The interaction effects between region and prehospital AAM on outcomes were similar to those of the entire analysis (Appendix 4). Therefore, the effects of bias due to variation in scene time interval were likely insignificant.

Other mechanisms behind this effect may include differences in the proficiency of providers in regional EMS systems. One reason for the various levels of proficiency in the performance of prehospital AAM across regional EMS systems is that the EMS providers in each system have different amounts of prehospital AAM-related experience. A recent meta-analysis reported that the success rate of ETI was associated with the number of ETIs previously performed by the EMS personnel. ${ }^{20}$ The authors also reported increased survival rates when providers had high exposure to ETI performance. In addition to the number of prehospital AAM cases, other factors associated with provider proficiency should be considered. As it is easier to perform SGA than ETI, proficiency in the former may not be a critical consideration. However, dislodging of the airway device is among the complications associated with SGA; thus, the concept of proficiency in AAM should be extended to post-procedural monitoring. Another unmeasured factor is teamwork among EMS providers during prehospital CPR. Previous studies have reported that teamwork during CPR is associated with the quality of CPR, including that of the chest compression fraction. ${ }^{21}$ Teamwork is also associated with the outcomes of patients with OHCA. ${ }^{22}$ An association between ETI and significant interruptions in chest compression performance has been demonstrated. ${ }^{23}$ Prehospital AAM without sufficient teamwork may contribute to increased rates of unnecessary interruption in chest compression performance.

This study has several limitations. First, we could not include data on the complications associated with prehospital AAM such as esophageal intubation. A recent RCT revealed that complications such as failure of ventilation and gastric regurgitation occurred significantly more frequently in the bag valve mask group than the ETI group. ${ }^{19}$ Second, no data on the prehospital AAM process were available in the PAROS dataset. The level of EMS providers involved in the performance of prehospital AAM and the number of attempts required for AAM insertion could influence the effects of prehospital AAM. Finally, residual bias may exist. It is challenging to determine whether the difference in the effects of prehospital AAM across the four cities was due to population characteristic-related differences (such as those pertaining to underlying disease) or differences in the EMS systems and prehospital care (resuscitation team and defibrillation energy), post-resuscitation care (targeted temperature management, percutaneous coronary intervention, and extracorporeal membrane 
oxygenation), and inhospital care (total CPR duration, length of intensive care unit stay, and number of ventilator-free days). However, we adjusted for the patients' demographic factors (such as age) and prehospital care provided (bystander CPR, initial ECG rhythm, etc.).

In summary, this study, which used data from an international database, showed that the effects of prehospital AAM on outcomes of OHCA patients differed according to variability in the individual EMS systems of the respective regions. To the best of our knowledge, our study is the first to assess regional variations in prehospital AAM and its effects across four Asian EMS systems.

\section{CONFLICT OF INTEREST}

No potential conflict of interest relevant to this article was reported.

\section{ACKNOWLEDGEMENTS}

The Korea Centers for Disease Control and Prevention financially supported this study (2012-E33010-00).

\section{REFERENCES}

1. Mozaffarian D, Benjamin EJ, Go AS, et al. Heart disease and stroke statistics: 2015 update: a report from the American Heart Association. Circulation 2015;131:e29-322.

2. Iwami $T$, Nichol G, Hiraide $A$, et al. Continuous improvements in "chain of survival" increased survival after out-of-hospital cardiac arrests: a large-scale population-based study. Circulation 2009;119:728-34.

3. Jeong S, Ahn KO, Shin SD. The role of prehospital advanced airway management on outcomes for out-of-hospital cardiac arrest patients: a meta-analysis. Am J Emerg Med 2016;34: 2101-6.

4. Fouche PF, Simpson PM, Bendall J, Thomas RE, Cone DC, Doi SA. Airways in out-of-hospital cardiac arrest: systematic review and meta-analysis. Prehosp Emerg Care 2014;18:244-56.

5. Beck B, Bray J, Cameron $P$, et al. Regional variation in the characteristics, incidence and outcomes of out-of-hospital cardiac arrest in Australia and New Zealand: results from the Aus-ROC Epistry. Resuscitation 2018;126:49-57.

6. Jacobs P, Grabinsky A. Advances in prehospital airway management. Int J Crit IIIn Inj Sci 2014;4:57-64.

7. Ong ME, Shin SD, Tanaka H, et al. Pan-Asian Resuscitation Outcomes Study (PAROS): rationale, methodology, and implementation. Acad Emerg Med 2011;18:890-7.
8. Shin SD, Ong ME, Tanaka $H$, et al. Comparison of emergency medical services systems across Pan-Asian countries: a Webbased survey. Prehosp Emerg Care 2012;16:477-96.

9. Rakun A, Allen J, Shahidah N, et al. Ethnic and neighborhood socioeconomic differences in incidence and survival from out of hospital cardiac arrest in Singapore. Prehosp Emerg Care 2019;23:619-30.

10. Kiyohara K, Sado J, Matsuyama T, et al. Out-of-hospital cardiac arrests during exercise among urban inhabitants in Japan: Insights from a population-based registry of Osaka City. Resuscitation 2017;117:14-7.

11. Lin $\mathrm{CH}, \mathrm{Ng} Y$, Chiang WC, et al. Variation of current protocols for managing out-of-hospital cardiac arrest in prehospital settings among Asian countries. J Formos Med Assoc 2016;115: 628-38.

12. Perkins GD, Jacobs IG, Nadkarni VM, et al. Cardiac arrest and cardiopulmonary resuscitation outcome reports: update of the Utstein Resuscitation Registry Templates for Out-of-Hospital Cardiac Arrest: a statement for healthcare professionals from a task force of the International Liaison Committee on Resuscitation (American Heart Association, European Resuscitation Council, Australian and New Zealand Council on Resuscitation, Heart and Stroke Foundation of Canada, InterAmerican Heart Foundation, Resuscitation Council of Southern Africa, Resuscitation Council of Asia); and the American Heart Association Emergency Cardiovascular Care Committee and the Council on Cardiopulmonary, Critical Care, Perioperative and Resuscitation. Circulation 2015;132:1286-300.

13. Edgren E, Hedstrand U, Kelsey S, Sutton-Tyrrell K, Safar P. Assessment of neurological prognosis in comatose survivors of cardiac arrest. BRCT I Study Group. Lancet 1994;343:1055-9.

14. McMullan J, Gerecht R, Bonomo J, et al. Airway management and out-of-hospital cardiac arrest outcome in the CARES registry. Resuscitation 2014;85:617-22.

15. Hasegawa $K$, Hiraide $A$, Chang $Y$, Brown DF. Association of prehospital advanced airway management with neurologic outcome and survival in patients with out-of-hospital cardiac arrest. JAMA 2013;309:257-66.

16. Shin SD, Ahn KO, Song KJ, Park CB, Lee EJ. Out-of-hospital airway management and cardiac arrest outcomes: a propensity score matched analysis. Resuscitation 2012;83:313-9.

17. White L, Melhuish T, Holyoak R, Ryan T, Kempton H, Vlok R. Advanced airway management in out of hospital cardiac arrest: A systematic review and meta-analysis. Am J Emerg Med 2018;36:2298-306.

18. Izawa J, Iwami T, Gibo K, et al. Timing of advanced airway management by emergency medical services personnel following 
out-of-hospital cardiac arrest: a population-based cohort study. Resuscitation 2018;128:16-23.

19. Jabre $P$, Penaloza $A$, Pinero $D$, et al. Effect of bag-mask ventilation vs endotracheal intubation during cardiopulmonary resuscitation on neurological outcome after out-of-hospital cardiorespiratory arrest: a randomized clinical trial. JAMA 2018;319:779-87.

20. Dyson K, Bray J, Smith K, Bernard S, Finn J. A systematic review of the effect of emergency medical service practitioners' experience and exposure to out-of-hospital cardiac arrest on patient survival and procedural performance. Resuscitation 2014;85:1134-41.
21. Yeung JH, Ong GJ, Davies RP, Gao F, Perkins GD. Factors affecting team leadership skills and their relationship with quality of cardiopulmonary resuscitation. Crit Care Med 2012;40: 2617-21.

22. Kim S, Ahn KO, Jeong S. The effect of team-based CPR on outcomes in out of hospital cardiac arrest patients: a meta-analysis. Am J Emerg Med 2018;36:248-52.

23. Wang HE, Simeone SJ, Weaver MD, Callaway CW. Interruptions in cardiopulmonary resuscitation from paramedic endotracheal intubation. Ann Emerg Med 2009;54:645-52. 
Appendix 1. Characteristics of patients with out-of-hospital cardiac arrest according to prehospital advanced airway use

\begin{tabular}{|c|c|c|c|c|}
\hline & \multicolumn{3}{|c|}{ EMS advanced airway management } & \multirow{2}{*}{ P-value } \\
\hline & All $(n=16,510)$ & No $(n=7,990)$ & Yes $(n=8,520)$ & \\
\hline Age $(y r)$ & & & & 0.34 \\
\hline $19-64$ & $4,982(30)$ & $2,472(31)$ & $2,510(29)$ & \\
\hline$\geq 65$ & $11,528(70)$ & $5,518(69)$ & $6,010(71)$ & \\
\hline Sex & & & & 0.23 \\
\hline Male & $10,266(62)$ & $4,967(62)$ & $5,299(62)$ & \\
\hline Female & $6,244(38)$ & $3,023(38)$ & $3,221(38)$ & \\
\hline Regional EMS system & & & & $<0.05$ \\
\hline Osaka & $8,055(49)$ & $2,821(35)$ & $5,234(61)$ & \\
\hline Seoul & $4,277(26)$ & $3,455(43)$ & $822(10)$ & \\
\hline Singapore & $2,046(12)$ & $308(4)$ & $1,738(20)$ & \\
\hline Taipei & $2,132(13)$ & $1,406(18)$ & $726(9)$ & \\
\hline Witnessed status & & & & $<0.05$ \\
\hline No & $10,000(61)$ & $5,040(63)$ & $4,960(58)$ & \\
\hline Yes & $6,510(39)$ & $2,950(37)$ & $3,560(42)$ & \\
\hline Bystander CPR & & & & $<0.05$ \\
\hline No & $10,208(62)$ & $5,011(63)$ & $5,197(61)$ & \\
\hline Yes & $6,302(38)$ & 2,979 (37) & $3,323(39)$ & \\
\hline Initial ECG rhythm & & & & $<0.05$ \\
\hline Shockable & $2,508(15)$ & $1,227(15)$ & 1,281 (15) & \\
\hline Non-shockable & $13,483(82)$ & $6,336(79)$ & 7,147 (84) & \\
\hline Unknown & $519(3)$ & $427(5)$ & $92(1)$ & \\
\hline EMS scene time interval & & & & $<0.05$ \\
\hline Missing & $343(2)$ & $135(2)$ & $208(2)$ & \\
\hline $0-8(\min )$ & $3,123(19)$ & $2,433(30)$ & $690(8)$ & \\
\hline $8-16$ (min) & $8,265(50)$ & $4,147(52)$ & $4,118(48)$ & \\
\hline $16-24(\min )$ & 4,038 (24) & $1,095(14)$ & $2,943(35)$ & \\
\hline 24-32 (min) & $741(4)$ & $180(2)$ & $561(7)$ & \\
\hline Median (IQR) & $12.75(9-17)$ & $10(7-14)$ & $15(11-18.6)$ & $<0.05$ \\
\hline \multicolumn{5}{|l|}{ Outcomes } \\
\hline Survival & $1,130(7)$ & 698 (8.7) & $432(5.1)$ & $<0.05$ \\
\hline Neurological recovery & $560(3)$ & $392(4.9)$ & $168(2.0)$ & $<0.05$ \\
\hline
\end{tabular}

Values are presented as number (\%) unless otherwise indicated.

EMS, emergency medical services; CPR, cardiopulmonary resuscitation; ECG, electrocardiography; IQR, interquartile range. 
Young Seok Oh, et al.

Appendix 2. Characteristics of patients with out-of-hospital cardiac arrest according to regional EMSs system

\begin{tabular}{|c|c|c|c|c|c|}
\hline & \multicolumn{4}{|c|}{ Regional EMS systems } & P-value \\
\hline Age $(\mathrm{yr})$ & & & & & $<0.05$ \\
\hline$\geq 65$ & $6,360(79.0)$ & $2,595(60.7)$ & $1,060(51.8)$ & $1,513(71.0)$ & \\
\hline Sex & & & & & $<0.05$ \\
\hline Male & $4,606(57.2)$ & $2,865(67.0)$ & $1,413(69.1)$ & $1,382(64.8)$ & \\
\hline EMS advanced airway & & & & & $<0.05$ \\
\hline No & $2,821(35.0)$ & $3,455(80.8)$ & $308(15.1)$ & $1,406(65.9)$ & \\
\hline Yes & $5,234(65.0)$ & $822(19.2)$ & $1,738(84.9)$ & $726(34.1)$ & \\
\hline Witnessed & & & & & $<0.05$ \\
\hline No & $5,140(63.8)$ & $2,256(52.7)$ & $951(46.5)$ & $1,653(77.5)$ & \\
\hline Initial ECG rhythm & & & & & $<0.05$ \\
\hline shockable & $868(10.8)$ & $905(21.2)$ & $482(23.6)$ & $253(11.9)$ & \\
\hline Non-shockable & $7,166(89.0)$ & $3,076(71.9)$ & $1,563(76.4)$ & $1,678(78.7)$ & \\
\hline Unknown & $21(0.3)$ & $296(6.9)$ & $1(0.0)$ & $201(9.4)$ & \\
\hline EMS scene time interval (min) & & & & & $<0.05$ \\
\hline Missing & 308 (3.8) & $14(0.3)$ & $4(0.2)$ & $17(0.8)$ & \\
\hline $0-8(\min )$ & $504(6.3)$ & $2,347(54.9)$ & $117(5.7)$ & $155(7.3)$ & \\
\hline $8-16(\min )$ & $4,033(6.3)$ & $1,710(54.9)$ & $1,266(5.7)$ & $1,256(7.3)$ & \\
\hline $16-24(\mathrm{~min})$ & $2,612(50.1)$ & $184(40.0)$ & $602(61.9)$ & $640(58.9)$ & \\
\hline $24-32(\min )$ & $598(32.4)$ & $22(4.3)$ & $57(29.4)$ & $64(30.0)$ & \\
\hline
\end{tabular}

Values are presented as number (\%) unless otherwise indicated.

EMS, emergency medical services; CPR, cardiopulmonary resuscitation; ECG, electrocardiography; IQR, interquartile range. 
Appendix 3. Multivariable analysis for the effects of prehospital advanced airway management and regional EMS systems on outcomes

\begin{tabular}{|c|c|c|c|c|c|c|}
\hline & \multirow{2}{*}{ Total } & \multirow{2}{*}{ Outcomes } & \multicolumn{2}{|c|}{ Unadjusted } & \multicolumn{2}{|c|}{ Adjusted } \\
\hline & & & $\mathrm{OR}$ & $95 \% \mathrm{Cl}$ & $\mathrm{OR}$ & $95 \% \mathrm{Cl}$ \\
\hline \multicolumn{7}{|c|}{ Neurological recovery } \\
\hline No & 7,990 & $392(4.9)$ & 1.00 & & 1.00 & \\
\hline Yes & 8,520 & $168(2.0)$ & 0.39 & $0.33-0.47$ & 0.30 & $0.24-0.38$ \\
\hline \multicolumn{7}{|c|}{ Regional EMS system ${ }^{b)}$} \\
\hline Taipei & 2,132 & $64(3.0)$ & 1.95 & $1.27-2.99$ & 1.96 & $1.21-3.18$ \\
\hline Osaka & 8,055 & $301(3.7)$ & 2.44 & $1.69-3.53$ & 3.95 & $2.64-5.90$ \\
\hline Seoul & 4,277 & $163(3.8)$ & 2.49 & $1.70-3.66$ & 1.00 & $0.64-1.56$ \\
\hline \multicolumn{7}{|c|}{ Survival to discharge } \\
\hline \multicolumn{7}{|c|}{ Prehospital advanced airway management $t^{\mathrm{a})}$} \\
\hline Taipei & 2,132 & $139(6.5)$ & 2.31 & $1.69-3.14$ & 3.42 & $2.42-4.84$ \\
\hline Osaka & 8,055 & $545(6.8)$ & 2.40 & $1.83-3.15$ & 4.51 & $3.36-6.05$ \\
\hline Seoul & 4,277 & $386(9.0)$ & 3.28 & $2.49-4.33$ & 2.24 & $1.63-3.09$ \\
\hline
\end{tabular}

Values are presented as number or number (\%).

EMS, emergency medical services; $\mathrm{OR}$, odd ratio; $\mathrm{Cl}$, confidence interval.

${ }^{a)}$ Adjusted to city, sex, age, witness arrest, bystander cardiopulmonary resuscitation, initial electrocardiogram, response time interval, and scene time interval. ${ }^{\text {b) }}$ Adjusted to EMS advanced airway management, sex, age, witness arrest, bystander cardiopulmonary resuscitation, initial electrocardiogram, response time interval, and scene time interval.

Appendix 4. Effects of prehospital advanced airway management on outcomes in interaction model with regional emergency medical service systems

\begin{tabular}{llll}
\hline Outcomes & & Adjusted OR & $95 \% \mathrm{Cl}$ \\
\hline $\begin{array}{l}\text { Neurological recovery } \\
\text { Interaction }\end{array}$ & AAM(-) & 1 & \\
Singapore & AAM(+) & 0.12 & $0.06-0.26$ \\
Taipei & AAM(+) & 0.58 & $0.31-1.10$ \\
Osaka & AAM(+) & 0.21 & $0.16-0.28$ \\
Seoul & AAM(+) & 0.79 & $0.52-1.20$ \\
Survival to discharge & & & \\
Interaction & AAM(-) & 1 & \\
Singapore & AAM(+) & 0.31 & $0.17-0.58$ \\
Taipei & AAM(+) & 1.04 & $0.69-1.55$ \\
Osaka & AAM(+) & 0.43 & $0.35-0.52$ \\
Seoul & AAM(+) & 0.99 & $0.75-1.30$ \\
\hline
\end{tabular}

$\mathrm{AAM}$, advanced airway management; $\mathrm{OR}$, odd ratio; $\mathrm{Cl}$, confidence interval. 